\title{
CliniProteus: A flexible clinical trials information management system
}

\author{
Venkatarajan S. Mathura ${ }^{1, *}$, Mahendiranath Rangareddy ${ }^{2}$, Pankaj Gupta $^{1}$, Michael Mullan ${ }^{1}$ \\ ${ }^{1}$ Roskamp Institute, Sarasota, Florida 34243, USA; ${ }^{2}$ Optimal solution and technologies Inc, Washington DC; \\ Venkatarajan S. Mathura* - E-mail: venkat@rfdn.org; Phone: 001-941-752-2949; * Corresponding author \\ received December 15, 2007; accepted December 18, 2007; published online December 28, 2007
}

\begin{abstract}
:
Clinical trials involve multi-site heterogeneous data generation with complex data input-formats and forms. The data should be captured and queried in an integrated fashion to facilitate further analysis. Electronic case-report forms (eCRF) are gaining popularity since it allows capture of clinical information in a rapid manner. We have designed and developed an XML based flexible clinical trials data management framework in .NET environment that can be used for efficient design and deployment of eCRFs to efficiently collate data and analyze information from multi-site clinical trials. The main components of our system include an XML form designer, a Patient registration eForm, reusable eForms, multiple-visit data capture and consolidated reports. A unique id is used for tracking the trial, site of occurrence, the patient and the year of recruitment.
\end{abstract}

Availability: http://www.rfdn.org/bioinfo/CTMS/ctms.html

Keywords: clinical trial; XML; NET; eCRF

Background:

Conducting clinical trials using defined protocols and reporting data regarding safety/efficacy is considered to be the bottle-neck in drug development process. Each trial requires several hundred pieces of data to be compiled at the end and further processed for statistical analysis. [1] A multi-site trial spans across several geographical locations with data integration and monitoring done at a single central location. Sophisticated software tools can help rapid data integration and monitoring of trial sites. Such software systems for managing clinical trials should be able to scaleup well with usage and at the same time should provide greater flexibility to incorporate changes for rapid development and deployment. [2] Each clinical trial is defined by a well characterized protocol that include questionnaires for inclusion and exclusion criteria, information regarding the participant, group assignment (either placebo or under treatment), adverse-event logs, sample information and laboratory results. A patient may be followed over several visits during which they may be on a specific drug. All these information is gathered in a case report form designed based on protocol requirements. The challenge of developing clinical trial software starts with transcribing case report forms into an electronic version with field labels such that data input can be mapped and extracted at later stage using unique identifiers. A flexible system should have a framework that can assemble information at several stages and provide a platform that can be used for coding CRFs. [3] Pharmaceutical and Drug Research organizations are extremely dependent on variety of information systems to support through their clinical trial ISSN 0973-2063

Bioinformation 2(3): 163-165 (2007) and drug discovery process. Organizations use variety of applications like Clinical Trial Management Software (CTMS), Electronic Data Capture (EDC) and other specialized clinical study solutions intended to reduce the time and cost associated with the drug discovery. Many applications available in the market are a stand alone solution developed to cater to either administrative or clinical research needs. In the clinical trial process, research, administration and compliance monitoring are interrelated and have lots of overlapping functionalities. Data is the integral part of the whole process and it is moved between discrete systems to support the clinical trial which in turn requires more time and resources to share information. In most of the organizations, more than one solution is required to complete the clinical trial process and there may be lot of integration bottlenecks, making the whole process complex and expensive. [4] Hence, an integrated solution with data capture, data management, workflow management, process integration and analytics is required to increase Return on Investments (ROI) at clinical research organizations. Also, clinical trial solution needs to be flexible and versatile to adapt to the ever growing needs of the clinical research community. CliniProteus is a scalable and flexible integrated solution intended to cater to the needs of the clinical research organization of any size. Our model can be extended easily to design flexible laboratory management system by modifying underlying XML templates. 


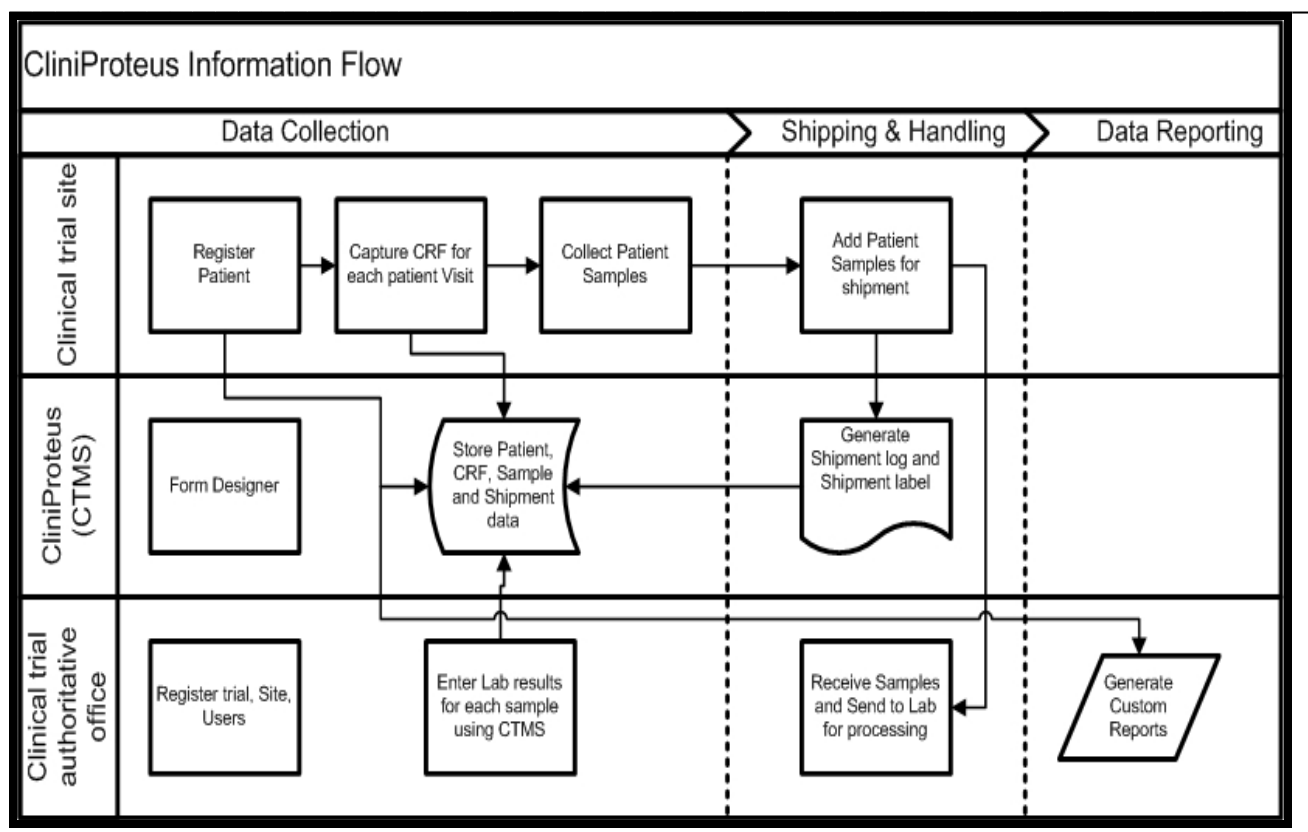

Figure 1: Information flow process in CliniProteus system

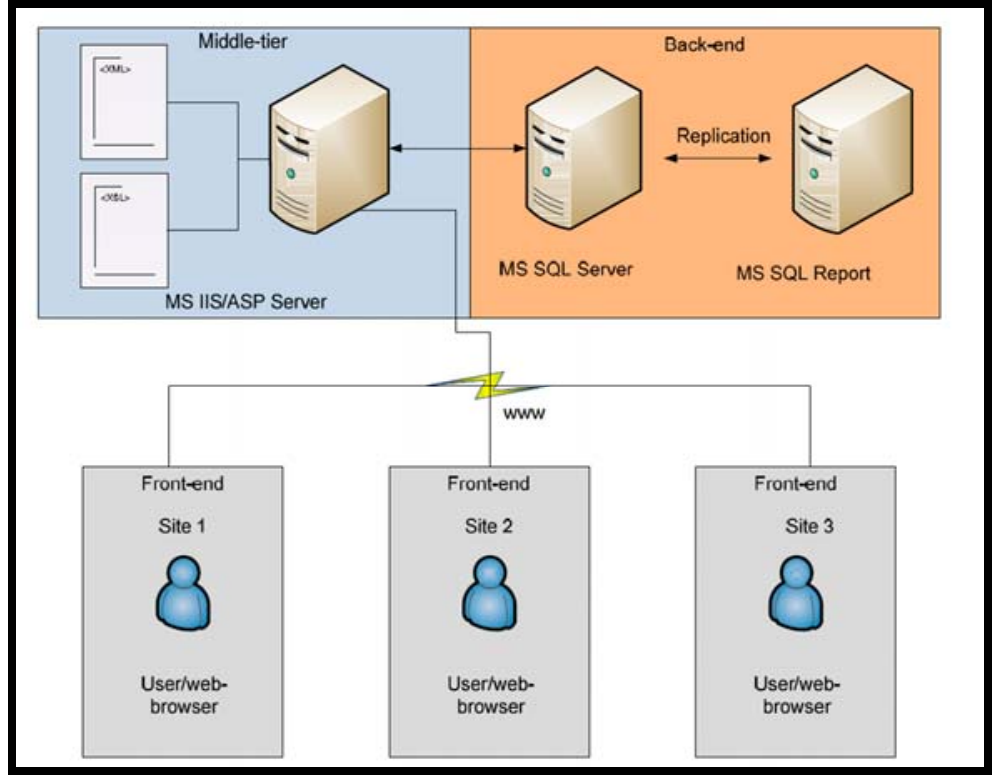

Figure 2: Multi-tier architecture of CliniProteus

\section{Approach:}

CliniProteus is an integrated software solution which includes and not limited to Clinical Trial Management System, logistics, Data Management, eCRF to capture data, and data analytics. CliniProteus is a truly flexible and scalable to include any new feature or functionality. The system currently has the following functionality to support clinical trials: (1) End User Administration; (2) Patient registration; (3) eCRF; (4) Verification of eCRF data entry; (5) Maintain inclusion/exclusion criteria, adverse event log;

ISSN 0973-2063

Bioinformation 2(3): 163-165 (2007)
(6) Visit management; (7) Shipping \& handling of Samples; (8) Clinical sample data integration; (9) Customized reports; (10) Research analytics.

In a typical clinical trial using CliniProteus, a patient is recruited and registered; for each of the recruited patients, electronic case report forms (eCRF) are used to collect information (vital statistics, adverse event, samples, concomitant medication etc) per visit. CliniProteus has a 


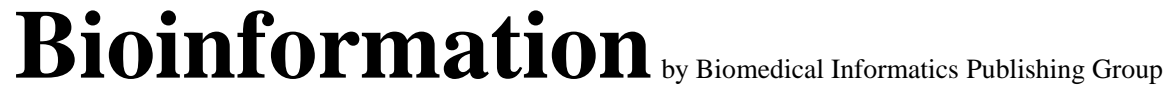

open access

\section{www.bioinformation.net}

\section{Software}

user friendly interface and displays only forms that are required to be filled out for each visit. Within the system multiple data entry and verification is available before locking forms for further modification.

Samples collected from patient during a visit are entered into CliniProteus. A user at trial site can then specify sample for the shipment when there are enough samples (batch shipment). Clinical trial center can receive the samples and sends it to the lab. The lab results are entered for each sample and stored in CliniProteus. The system automatically integrates information avoiding end-user from complicated data tracking tasks. Using CliniProteus researchers can track patient visits, collect metrics to support clinical trial, generate adverse event logs, and generate CRF for submission and other custom generated reports. Any changes to the data are continuously monitored and recorded. The system provides strict hierarchical controls and adheres to HIPAA standards.

\section{Design and technology used:}

CliniProteus is a web based solution developed using the Microsoft .NET platform. [5] It uses Microsoft.NET 2.0, Windows Server 2003 with IIS 6.0, SQL Server 2005, SQL Server 2005 Reporting Services, SQL and SQL Server 2005 Analysis Services. [6] It uses XML and XSLT technology to provide easy scalability and data integrity.

CliniProteus eCRF forms used by the investigators to collect information per visit were developed using XML as the template language and XSLT as transforming tool in ASP.NET environment. Investigator pulls out the form on the internet, fills all the patient visit information, and hits the save button to save all the information into a SQL Server table. The eCRF data is stored as XML in the database and it leverages on the new SQL Server 2005 XML data type to provide robust data integrity using a simple design. SQL Server 2005 supports XQuery, any specific data in an XML document stored in the database table can be easily retrieved using a RDBMS SQL query by embedding XQuery into it. Logistics module was built to track the samples collected from the patients, and its results from the laboratory analysis. Sample data is tightly integrated with the clinical data collected using the eCRF forms. SQL Server 2005 Reporting Services is used to generate and publish custom reports with ease. SQL Server
2005 Analysis Services is used to analyze data and generate analytic presentations like cubes, graphs etc.

\section{Output:}

CliniProteus can be used to register patient information, fill eCRF, query patient records and status. Users can customize reports using MS SQL Report services to cater to their research needs. New forms or fields can be deployed rapidly by amending existing XML or new XML schema for an underlying form. Since the data is stored as $\mathrm{XML}$, one can use XML tools to migrate or combine XMLs to create new structured data file.

\section{Caveat:}

We have not implemented automatic data validation for individual fields. This will be a major draw back for this version that will be corrected in the future version. The system uses native .NET components that will restrict operating system independence.

\section{Future development:}

CliniProteus is a living solution that will be continuously enhanced to suit the changing needs of business and technology. Few of the enhancement that are planned in the near term are to build an automated notification system to send email for an adverse event or for any custom defined notification rules, provide a scheduling system to facilitate patient visit, use of SQL Server 2005 data mining features to perform data retrieval and evaluation, and to provide drill through reports.

\section{Acknowledgement:}

We thank Roskamp Foundation for the financial support for this work.

\section{References:}

[01] C. Estellat, et al., Contemp Clin Trials, (2007) [PMID: 17974503]

[02] M. G. Aspinall and R. G. Hamermesh, Harv Bus Rev., 85: 108 (2007) [PMID: 17972499]

[03] J. D. Rademacher and S. Lippke, Behav Res Methods, 39: 415 (2007) [PMID: 17958153]

[04] H. Shi and L. Weiler, J BMC Med Inform Decis Mak., 7: 23 (2007) [PMID: 17697328]

[05] http://www.asp.net

[06] http://www.microsoft.com

Edited by P. Kangueane

Citation: Mathura et al., Bioinformation 2(4): 163-165 (2007)

License statement: This is an open-access article, which permits unrestricted use, distribution, and reproduction in any medium, for non-commercial purposes, provided the original author and source are credited. 